\title{
A Proposal for Long-Term Missions of Small Lunar Rovers Using an MLI Curtain System
}

\author{
Jang-Joon Lee $\mathbb{D}^{1,2}$ and Kyu-Hong Kim $\mathbb{D}^{1}$ \\ ${ }^{1}$ Hypersonic \& Rarefied Flow Laboratory, Department of Aerospace Engineering, Seoul National University, 1 Gwanak-ro, \\ Gwanak-gu, Seoul 08826, Republic of Korea \\ ${ }^{2}$ Korea Aerospace Research Institute (KARI), 169-84, Gwahak-ro, Yuseong-gu, Daejeon 34133, Republic of Korea
}

Correspondence should be addressed to Kyu-Hong Kim; aerocfd1@snu.ac.kr

Received 18 March 2019; Accepted 29 May 2019; Published 2 July 2019

Academic Editor: Yue Wang

Copyright ( $) 2019$ Jang-Joon Lee and Kyu-Hong Kim. This is an open access article distributed under the Creative Commons Attribution License, which permits unrestricted use, distribution, and reproduction in any medium, provided the original work is properly cited.

\begin{abstract}
This paper proposes a lunar night survival method for small rovers using an MLI (Multilayer Insulation) curtain system for longterm missions. Until recently, it was difficult to install RHU (Radioisotope Heating Units) or other temperature maintenance devices on small lunar rovers to enable lunar night survival, and so such rovers could only perform short two-week missions. Thermal analysis results show that small rovers could survive during lunar nights by moving into a shelter located inside the MLI curtain of the lander without mounting temperature maintenance devices. In order to enhance the feasibility of the MLI curtain system, we also propose ideas of a double-layer MLI and a rover configuration without solar cells.
\end{abstract}

\section{Introduction}

Lunar landers and rovers that perform missions exceeding a month (one lunar day) are required to survive in a temperature environment of $-190^{\circ} \mathrm{C}$ for two weeks without any supply of heat from external heat sources such as solar heat. As lunar landers or rovers cannot survive without a heat source during this period, most lunar exploration programs employ RHU (Radioisotope Heating Units) or RTG (Radioisotope Thermoelectric Generators) [1] for lunar night survival, as described in Table 1.

With concerns of RHU and RTG regarding radioactive contamination, several efforts are being made to find other methods for lunar night survival. Balasubramaniam et al. [2] proposed the Thermal Wadis to survive lunar nights without RHU or RTG. Thermal Wadis is a device that maintains the temperature of rovers during lunar nights by absorbing heat from the sun during the daytime of the Moon by installing an object of high capacity and low thermal conductivity on the surface of the Moon. Thermal Wadis accumulates solar heat during the daytime of the Moon and employs heat shielding to limit heat radiation to space during the lunar night. The difficulty of this method involves the requirement of complex and heavy systems to implement Wadis.

Notsu et al. [3] proposed a heat exchange device with regolith for night survival. Considering that temperature swings are remarkably small during the daytime and nighttime periods at depths of only $1 \mathrm{~m}$ below the surface of the Moon, the device penetrates the surface of the ground below the lunar lander to enable night survival through heat exchange with the regolith. This proposal should identify feasible methods for penetrating the string through the lunar surface.

As of the time of this writing, survival methods for lunar nights that have been successfully proven still use RHU or RTG systems. Table 1 summarizes the specifications of unmanned rovers and landers, not including US manned mission, to compare with the subject of this study. Table 1 shows that all past unmanned lunar surface exploration missions involved the use of RHU or RTG systems.

The subject of this study is a small rover with a mass range of $20 \sim 30 \mathrm{~kg}$. For small lander and rover programs, it is difficult to set up an RHU or RTG system in small rovers. For this reason, small rovers were not able to survive lunar 
TABLE 1: Specification of unmanned lunar rovers.

\begin{tabular}{lccccc}
\hline $\begin{array}{l}\text { Project title } \\
\text { (spacecraft/rover) }\end{array}$ & $\begin{array}{c}\text { Luna 17/Lunokhod 1 } \\
{[22]}\end{array}$ & $\begin{array}{c}\text { Luna 21/Lunokhod 2 } \\
{[22]}\end{array}$ & $\begin{array}{c}\text { Chang'e-3/Yutu } \\
{[22]}\end{array}$ & $\begin{array}{c}\text { Chang'e-4/Yutu } \\
2\end{array}$ & $\begin{array}{c}\text { Chandrayaan-2 } \\
{[4]}\end{array}$ \\
\hline Mission date & $1970.11 \sim 1971.9$ & $1973.1 \sim 1973.5$ & $2013.12 \sim 2016.8$ & $2019.1 \sim$ & 2019.07 \\
Lander dry mass & $\mathrm{N} / \mathrm{A}$ & $1814 \mathrm{~kg}$ & $1200 \mathrm{~kg}$ & $1200 \mathrm{~kg}[23]$ & $1471 \mathrm{~kg}$ \\
Rover mass & $756 \mathrm{~kg}[7]$ & $840 \mathrm{~kg}$ & $120 \mathrm{~kg}$ & $140 \mathrm{~kg}[23]$ & $27 \mathrm{~kg}$ \\
Landing site & $38^{\circ} \mathrm{N} 35^{\circ} \mathrm{W}$ & $26^{\circ} \mathrm{N} 30^{\circ} \mathrm{E}$ & $44^{\circ} \mathrm{N} 20^{\circ} \mathrm{W}$ & $46^{\circ} \mathrm{S} 178^{\circ} \mathrm{E}[24]$ & $71^{\circ} \mathrm{S} 23^{\circ} \mathrm{E}[25]$ \\
$\begin{array}{l}\text { Country } \\
\text { Survival method of the } \\
\text { rover during lunar nights }\end{array}$ & $\mathrm{USSR}$ & $\mathrm{USSR}$ & $\mathrm{China}$ & $\mathrm{China}$ & India \\
\hline
\end{tabular}

nights until recently. As shown in Table 1, rovers that perform long-term missions of more than two weeks weigh at least $100 \mathrm{~kg}$, and the $27 \mathrm{~kg}$ small rover that was loaded in Chandrayaan-2 [4] was designed for short-term missions of less than two weeks.

This study proposes a lunar night survival method involving an MLI curtain system that enables small rovers of $20 \sim 30 \mathrm{~kg}$ to perform long-term missions. The system can be summarized as a small rover without RHUs that performs mission tasks during the daytime and continues to survive in an MLI curtain shelter on the lunar lander during lunar nights. The rover can perform short missions even during lunar nights according to power margins, and it can perform missions again upon the return of the lunar day. In order to prove the feasibility of the MLI curtain system for lunar night survival, this study introduces a thermal model of a lunar lander and a rover in a lunar thermal environment and presents the analysis results.

\section{Concept Idea of the MLI Curtain System for Long-Term Missions of Small Rovers}

Okishio et al. [5] proposed the MLI skirt as an auxiliary means in addition to strings for heat exchange with lunar surface regolith for lunar night survival. The basic concept of this study is similar to the MLI skirt as a shelter for a small rover for lunar night survival. However, unlike the skirt introduced in the study by Okishio et al. [5], the MLI is configured in a curtain shape to allow small rovers to freely enter and exit the curtain. Figure 1 shows a conceptual configuration of the MLI curtain system for a small rover.

The shape of the MLI curtain proposed in this study is similar to vertical blind curtains used in a typical home. The system has the advantage of allowing the rover to pass through the MLI curtain without assistance from any special opening/closing mechanisms. As shown in Figure 1(a), a rover can survive in a shelter during lunar nights and move out from the shelter through the MLI curtain on its own during the lunar daytime, as shown in Figure 1(b).

In this study, the temperature of the lander during lunar nights can be maintained using RHUs and a lid. Park et al. [6] introduced a lunar lander thermal model and presented analysis results for lunar night survival. According to their results, the combination of a lid and RHUs was more effective compared to other thermal control devices such as louvers or heat switches. The combination of a lid and RHUs was implemented in the Lunokhod series [7], which were lunar exploration rovers of the former Soviet Union.

From a thermal control perspective, RHUs have the disadvantage of generating heat not only during lunar nights but also during lunar daytime. Although RTGs can produce electrical power even without sunlight, such devices have large volumes and significant weights. Considering the case of the Galileo probe [8], which employed an RTG that weighed up to $56 \mathrm{~kg}$ with a capacity of $300 \mathrm{~W}$, RHUs are more suitable for lunar night survival compared to RTGs for small landers.

\section{Thermal Modeling}

3.1. Thermal Modeling of Lunar Regolith and Verification. Among the studies involving the thermal modeling of lunar regolith and the prediction of lunar surface temperatures by Vasavada et al. [9], Hager [10], and Christie et al. [11], this study used the thermal modeling method proposed by Christie et al. [11]. Of the various thermal modeling variables used by Christie et al. [11], only Moon albedo was quoted by Vasavada et al.'s study [12] based on LRO (Lunar Reconnaissance Orbiter) measurement results. The main variables used for lunar regolith thermal modeling are shown in Table 2.

"Fluff" in Table 2 is a term referring to a surface layer up to a depth of $0.02 \mathrm{~m}$ from the surface of the Moon, and "regolith" is a deeper layer. In other sections of this paper, "regolith" will refer to a combination of fluff and regolith. The thickness of lunar regolith was modeled as $0.6 \mathrm{~m}$. Figure 2 shows the result of modeling lunar regolith. The modeling and analysis of the lunar regolith were conducted using Thermal Desktop and Sinda-Fluint [13]. The central part of the lunar regolith model was further subdivided as the lunar lander and the rover are located at the corresponding location. This ensures that the radiative heat exchange between the lunar surface and the lunar lander and the rover further improves the reliability of the analysis.

In order to verify the lunar regolith thermal model, we used the LRO lunar surface temperature measurement data from Williams et al. [14] as a reference. The reference data values and analysis results are shown in Figure 3. In Figure 3, the $x$-axis is the angle from the Sun to the Moon, 


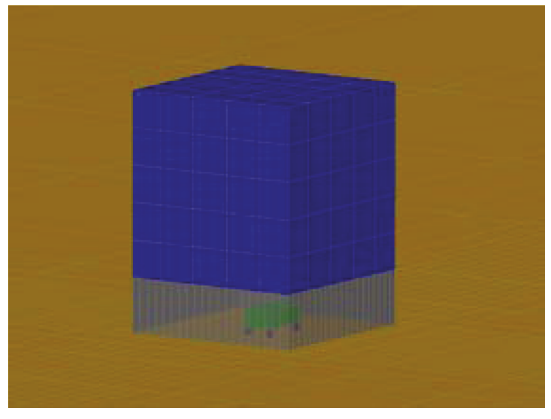

(a) A rover in a shelter

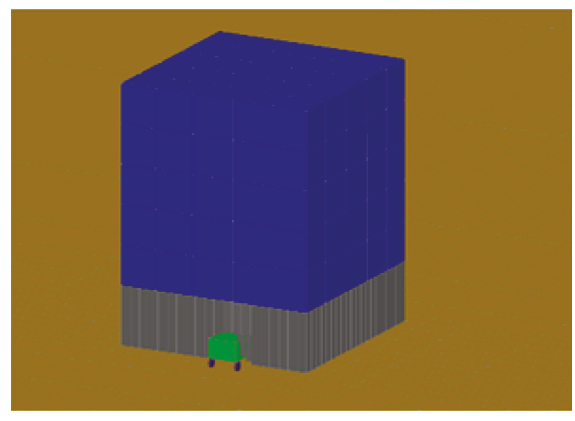

(b) A rover moving outside

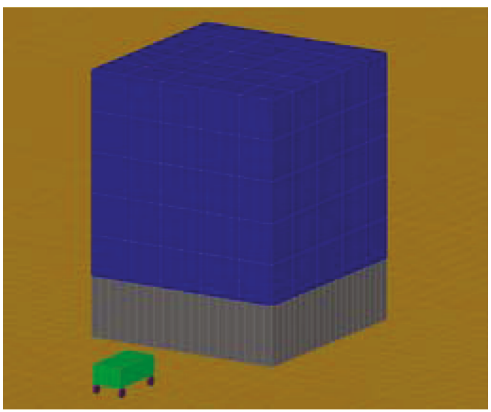

(c) A rover outside

Figure 1: MLI curtain system for the survival of a rover during lunar nights.

TABLE 2: Thermal model variables for lunar surface modeling.

\begin{tabular}{lc}
\hline Modeling variables & Value \\
\hline $\begin{array}{l}\text { Moon surface IR } \\
\text { emissivity }\end{array}$ & 0.97 \\
$\begin{array}{l}\text { Moon albedo [12] } \\
\text { Density of fluff }\left(\mathrm{kg} / \mathrm{m}^{3}\right)\end{array}$ & $A(\theta)=0.08+0.045(\theta / 45)^{3}+0.14(\theta / 90)^{8}$ \\
$\begin{array}{l}\text { Conductivity of fluff } \\
(\mathrm{W} / \mathrm{m} / \mathrm{K})\end{array}$ & 1000 \\
$\begin{array}{l}\text { Density of regolith } \\
\left(\mathrm{kg} / \mathrm{m}^{3}\right)\end{array}$ & $9.22 E-4\left[1+1.48(T / 350)^{3}\right]$ \\
$\begin{array}{l}\text { Conductivity of } \\
\text { regolith }(\mathrm{W} / \mathrm{m} / \mathrm{K})\end{array}$ & 2000 \\
$\begin{array}{l}\text { Specific heat of fluff } \\
\text { and regolith }(\mathrm{Jg} / \mathrm{k})\end{array}$ & $9.30 E-3\left[1+0.73(T / 350)^{3}\right]$ \\
$\begin{array}{l}\text { Imposed heat flow } \\
\left(\mathrm{W} / \mathrm{m}^{2}\right)\end{array}$ & 0.031 \\
\hline
\end{tabular}

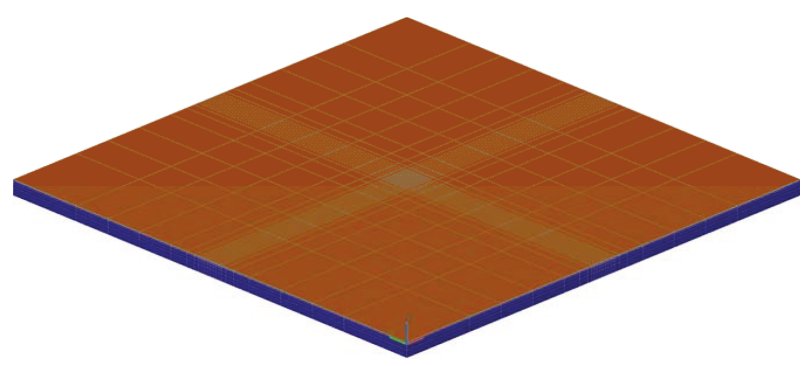

Figure 2: Thermal model of lunar regolith. and the $y$-axis is the temperature of the lunar surface with units in absolute temperature $(\mathrm{K})$.

Figure 3 shows that the analysis results of the lunar surface temperature were similar to the LRO measurement data for the latitude $0^{\circ}$ and latitude $30^{\circ}$ cases. On the other hand, in the case of latitude $60^{\circ}$ and latitude $85^{\circ}$, there were slight deviations on the cold side of the Moon as the modeling variables presented in the study by Christie et al. [11] were values derived by approximation for all positions in lunar surface modeling. Considering that the landing sites of lunar landers launched to date were mostly between latitude $0^{\circ}$ and latitude $\pm 40^{\circ}$ [15], in addition to the fact that the landing position of the lunar lander and the rover in this study is also latitude $0^{\circ}$, Figure 3 shows that the proposed regolith modeling possesses sufficient accuracy to be utilized here. In this study, the landing position of the lunar lander and the rover was set to latitude $0^{\circ}$ as the position of latitude $0^{\circ}$ is the worst from a thermal perspective due to having the largest temperature difference between day and night on the lunar surface. This follows the basic rules of thermal design/analysis to confirm the survivability of landers and rovers even under the worst thermal conditions [16].

3.2. Thermal Modeling of the Lunar Lander and the Rover. With the purpose of this study being to review a lunar night survival method for a small lunar rover, the thermal model of the lunar lander and the rover was made as simple as possible.

Table 3 shows the specifications and temperature requirements of the small lunar lander and the rover that were assumed in this study. The numerical values for the lunar lander in Table 3 are similar to those assumed by Park et al. [6]. 


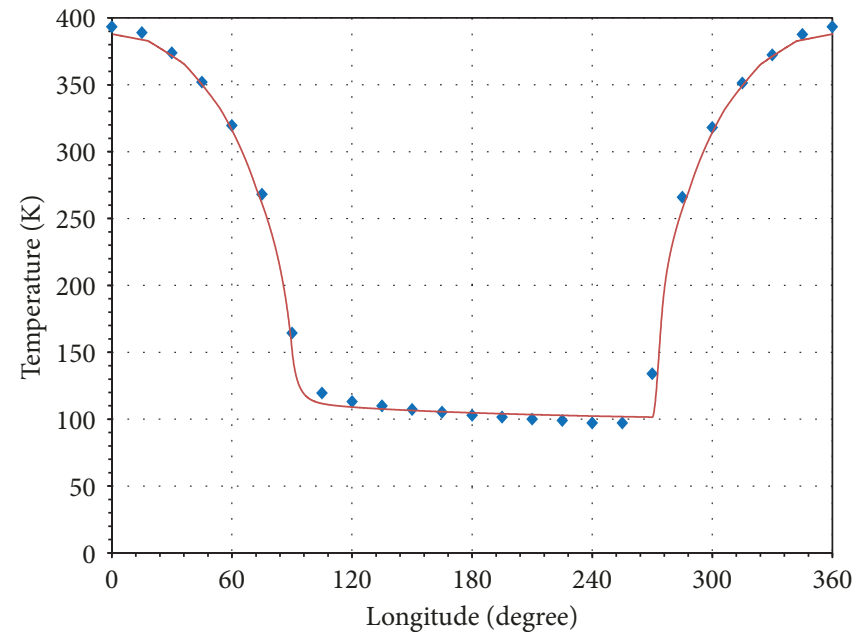

- Latitude 0

- Reference

(a) Result at latitude $0^{\circ}$

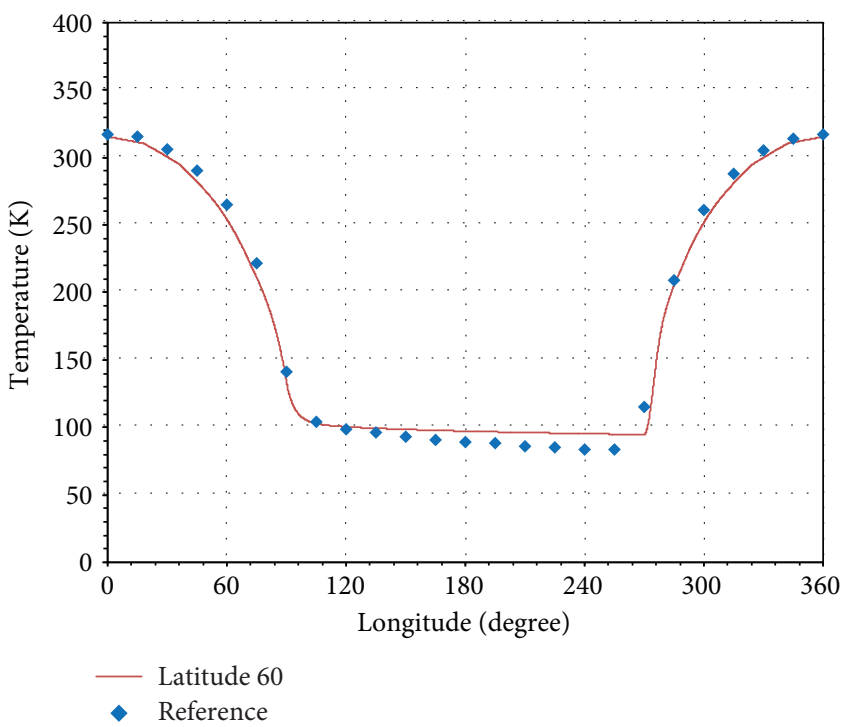

(c) Result at latitude $60^{\circ}$

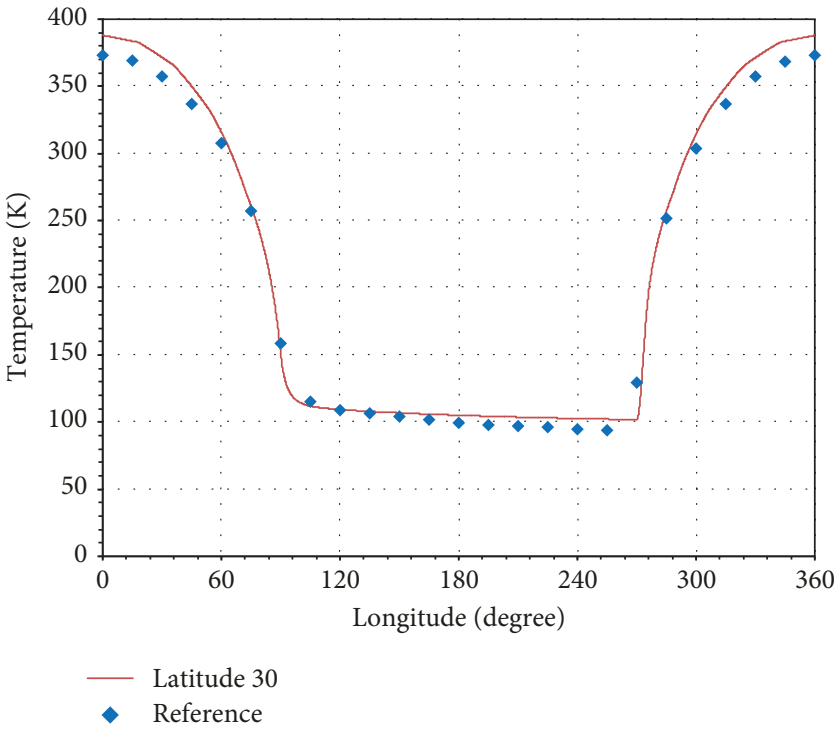

(b) Result at latitude $30^{\circ}$

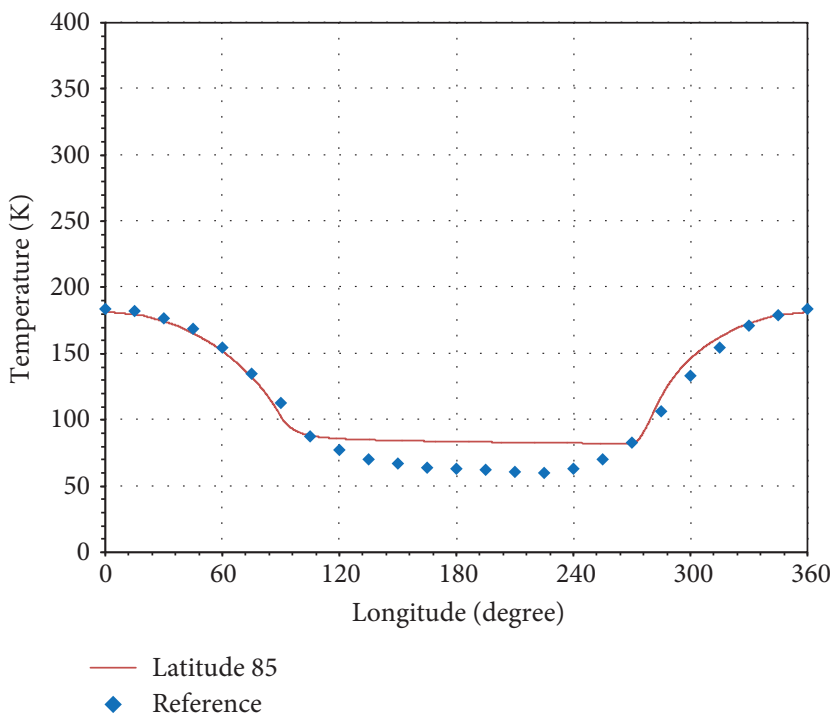

(d) Result at latitude $85^{\circ}$

FiguRE 3: Temperature calculation results of the lunar surface at various latitudes.

The thermal model of the lunar lander was designed as a cubic shape consisting of 125 nodes with the width, length, and height being five nodes long. The thermal conductivity of the lander model was assumed as $20 \mathrm{~W} / \mathrm{m} / \mathrm{K}$, which is $1 / 8$ of the thermal conductivity of general aluminum, instead of the internal radiation heat exchange in the empty space inside the lunar lander. Table 3 shows that the heat dissipation of the lunar lander was $120 \mathrm{~W}$ during the day and $10 \mathrm{~W}$ during the night as the heat dissipation of the lunar lander was assumed as shown in Table 4.

As the average power consumption during a lunar night was assumed as $10 \mathrm{~W}$ in Table 4 , the battery capacity of the lunar lander should be at least $3600 \mathrm{Wh}(=15$ days $\times 24$ hours/day $\times 10 \mathrm{~W}$ ) for lunar night survival. In this case, assuming the typical specific energy of a battery as $145 \mathrm{Wh} / \mathrm{kg}$ [17], the minimum mass of the required battery is calculated as $24.8 \mathrm{~kg}$. With the mass of the lunar lander assumed in this study being $300 \mathrm{~kg}$, the mass of the battery is approximately $8 \%$ of the total dry mass. Considering that the mass ratio of the battery in the Apollo lunar landing mission was approximately $1 \%$ [18], the battery mass ratio of this study is not a small value.

As the assumed mass of the battery of this study was already high, it was difficult to increase the capacity of the battery to consume heater power during lunar nights. Therefore, it was concluded that the supply of the heat source for lunar night survival should solely depend on the RHU. If heater power is consumed for lunar night survival, an incremental increase in battery capacity of $360 \mathrm{Wh}$ is required for the consumption of $1 \mathrm{~W}$ of heater power, which results in a $2.5 \mathrm{~kg}$ mass increase per watt. Such effects make it increasingly difficult to meet lander mass requirements. 
TABLE 3: Assumed specifications of the lunar lander and the rover.

\begin{tabular}{lc}
\hline Lunar lander & \\
Dry mass & $300 \mathrm{~kg}$ \\
Size $(\mathrm{L} \cdot \mathrm{W} \cdot \mathrm{H})$ & $1 \mathrm{~m} \times 1 \mathrm{~m} \times 1 \mathrm{~m}$ \\
Specific heat & $900 \mathrm{~kg} / \mathrm{J} / \mathrm{K}$ \\
Conductivity & $20 \mathrm{~W} / \mathrm{m} / \mathrm{K}$ \\
Heat dissipation (day/night) & $120 \mathrm{~W} / 10 \mathrm{~W}$ \\
Operating temperature range & $-20^{\circ} \mathrm{C} \sim 50^{\circ} \mathrm{C}$ \\
\hline Rover & \\
Mass & $25 \mathrm{~kg}$ \\
Size $(\mathrm{L} \cdot \mathrm{W} \cdot \mathrm{H})$ & $0.4 \mathrm{~m} \times 0.25 \mathrm{~m} \times 0.15 \mathrm{~m}$ \\
Specific heat & $900 \mathrm{~kg} / \mathrm{J} / \mathrm{K}$ \\
Heat dissipation (day/night) & $15 \mathrm{~W} / 0.5 \mathrm{~W}$ \\
Operating temperature range & $-15^{\circ} \mathrm{C} \sim 45^{\circ} \mathrm{C}$ \\
\hline
\end{tabular}

TABle 4: Assumed power consumption of the lunar lander.

\begin{tabular}{lcc}
\hline Unit & $\begin{array}{c}\text { Day } \\
\text { (mission profile) }\end{array}$ & $\begin{array}{c}\text { Night } \\
\text { (sleep mode) }\end{array}$ \\
\hline Main computer & $40 \mathrm{~W}$ & $5 \mathrm{~W}$ \\
Power converter \& distributer & $40 \mathrm{~W}$ & $3 \mathrm{~W}$ \\
Communication transponder & $20 \mathrm{~W}$ & $1 \mathrm{~W}$ \\
Battery & $0 \mathrm{~W}$ & $1 \mathrm{~W}$ \\
Location \& attitude sensors & $5 \mathrm{~W}$ & $0 \mathrm{~W}$ \\
Lander payloads & $15 \mathrm{~W}$ & $0 \mathrm{~W}$ \\
Total & $120 \mathrm{~W}$ & $10 \mathrm{~W}$ \\
\hline
\end{tabular}

Figure 4 shows the thermal model of the lunar lander and the rover of this study. Thermal Desktop and Sinda-Fluint [13] were used for thermal modeling and analysis of the lunar lander and the rover as well as the land regolith.

As illustrated in Figure 4(a), the lunar lander was modeled as a $1 \mathrm{~m} \times 1 \mathrm{~m} \times 1 \mathrm{~m}$ cube, and the lunar surface was modeled as a $20 \mathrm{~m} \times 20 \mathrm{~m}$ square as in Okishio et al.'s [5] study. In order to improve the accuracy of the analysis, the node size of the lunar surface in the vicinity of the lunar lander was smaller compared to nodes located further away from the lander. As shown in Figure 4(b), the lunar lander was designed as a cubic model with a total of 125 nodes. In order to efficiently radiate heat without interfering with the surrounding surface temperature, a radiator was installed in the direction of deep space.

As shown in Table 3, the rover was modeled as a single node that was $40 \mathrm{~cm} \times 25 \mathrm{~cm} \times 15 \mathrm{~cm}$ in size with a mass of $25 \mathrm{~kg}$. The power consumption of the rover was assumed as $15 \mathrm{~W}$ during lunar day missions and $0.5 \mathrm{~W}$ standby power during lunar nights. The standby power of $0.5 \mathrm{~W}$ while the rover hibernates inside the MLI curtain during lunar nights means that an additional $1.25 \mathrm{~kg}$ of battery mass is required in a similar manner to the situation described in the previous section. As such, it could be concluded that it is important to minimize the standby power of the rover in order to meet the mass requirements of the rover. If meeting the mass requirements of the rover proves challenging, the rover could be charged by the lander during lunar nights, which is an idea further described in Section 5.

In this study, the model of the MLI uses the CR-model (conduction-radiation model) introduced by Krishnaprakas et al. [19], which is given by the following equation.

$$
Q\left(\mathrm{~W} / \mathrm{cm}^{2}\right)=\mathrm{h}^{\prime}\left(T_{\mathrm{h}}-T_{\mathrm{c}}\right)+\mathcal{E}_{\text {eff }}^{\prime} \sigma\left(\mathrm{T}_{\mathrm{h}}{ }^{4}-\mathrm{T}_{\mathrm{C}}{ }^{4}\right) .
$$

In equation (1), $Q$ represents the heat flow rate through the MLI. The MLI specification is assumed to be 10 single spacer layers, the value of $h$ is $7.92 \mathrm{E}-07\left(\mathrm{~W} / \mathrm{cm}^{2} \cdot \mathrm{K}\right)$, the value of $\varepsilon^{\prime}$ eff is $3.27 E-03$, and $\sigma$ is the Stefan-Boltzmann constant. The values of $T_{\mathrm{h}}$ and $T_{\mathrm{c}}$ in equation (1) represent the temperatures at both sides of the MLI.

In this study, the radiator optical properties of the lander and the rover were 0.06 for solar absorption and 0.83 for infrared emissivity, as with the example of the UV reflective-coated OSR (Optical Solar Reflector) of Qioptiq [20]. The bottom surface coating of the lunar lander was designed with black paint to enhance heat exchange with the rover to enhance lunar night survival.

\section{Thermal Analysis}

4.1. Thermal Analysis Case. In this study, the lunar exploration rover performs its missions outside the lunar lander during the lunar daytime, and moves back into the lunar lander MLI curtain during the lunar nighttime to survive, as described in the previous section. In addition, the lunar lander covers the radiator with a lid, which is a panel equipped with solar cells during lunar nights, similar to Lunokhod [7]. During lunar nights, the lander solely uses RHUs as a heat source instead of an electrical heater. Figure 5(a) shows that the rover performs its missions outside the lunar lander, during the lunar daytime. In addition, the solar panel of the lander is deployed. Figure 5(b) shows that the rover moves into the MLI curtain of the lunar lander, and the solar panel that acts as a lid covers the radiator to prevent heat loss. As it is required for the solar panel, which acts as a lid, to cover the radiator and perform heat shielding during lunar nights, the design should adopt the application of the MLI to the rear end of the solar panel. The heat dissipation of the lander and the rover for one lunar day is shown in Table 3.

The landing position of the lunar lander was set as the latitude $0^{\circ}$ position of the Moon, where the temperature variation of the lunar surface is the most extreme. The solar heat flux was assumed as $1366 \mathrm{~W} / \mathrm{m}^{2}$. In Figure 6, the positions of the lander and the rover at sunrise, noon, sunset, and midnight during a lunar day are shown through a concept diagram.

4.2. Design of the Radiator Area and RHU through Thermal Analysis. As described in the previous section, in order for a small lunar lander to survive in the cryogenic environment of lunar nights, it is efficient to supply heat through RHUs. From a thermal control perspective, RHUs have the disadvantage of continually dissipating heat during lunar days in addition to lunar nights. Therefore, the lunar lander should 


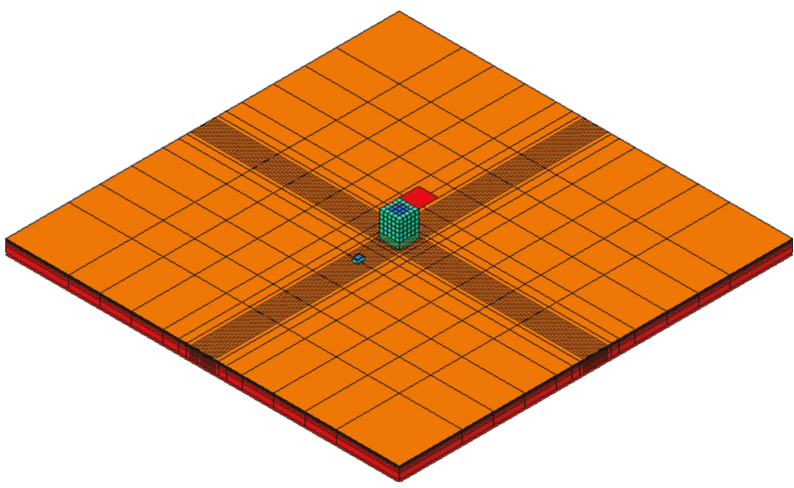

(a) Overall thermal model

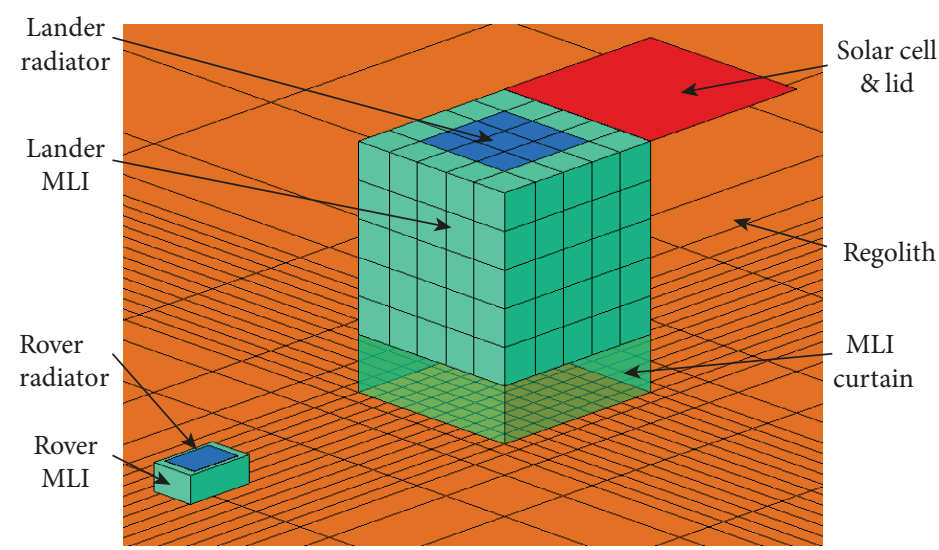

(b) Modeling of the lander and the rover

Figure 4: Thermal model of the regolith, the lander, and the rover.

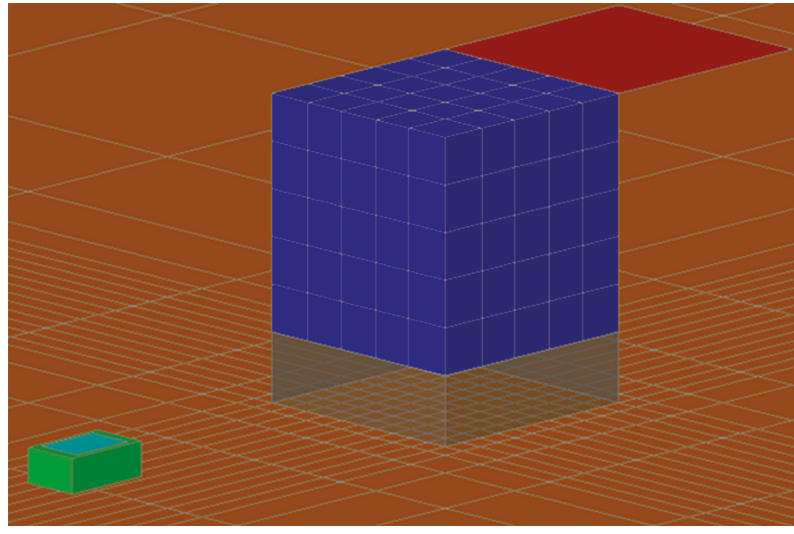

(a) Configuration during a lunar day

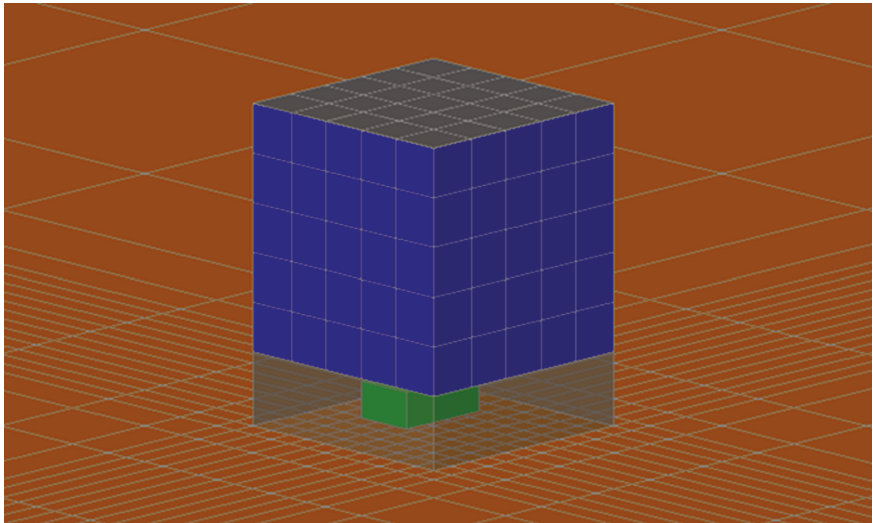

(b) Configuration during a lunar night

Figure 5: Mission configuration of a lunar lander and a rover.

radiate the heat generated by the RHUs in addition to the heat of the lunar lander itself, and the heat dissipation area should be designed accordingly.

The procedure of designing the radiator area and the capacity of the RHU of a lunar lander is as follows. First, the radiator area of the lunar lander should be designed so that the temperature of the lunar lander does not exceed the maximum acceptance temperature during lunar days under the thermal environment described in Table 3 . If the lunar lander has no RHU, the temperature of the lunar lander may fall below the minimum acceptance temperature during lunar nights. The design should calculate the proper number of RHUs that prevents the temperature of the lunar lander from falling below the minimum acceptance temperature. Due to the additional RHU heat, the lander temperature rises to higher temperatures during lunar days. Hence, a larger radiator area is required, which should be recalculated. As the lid covers the radiator area during lunar nights, the 


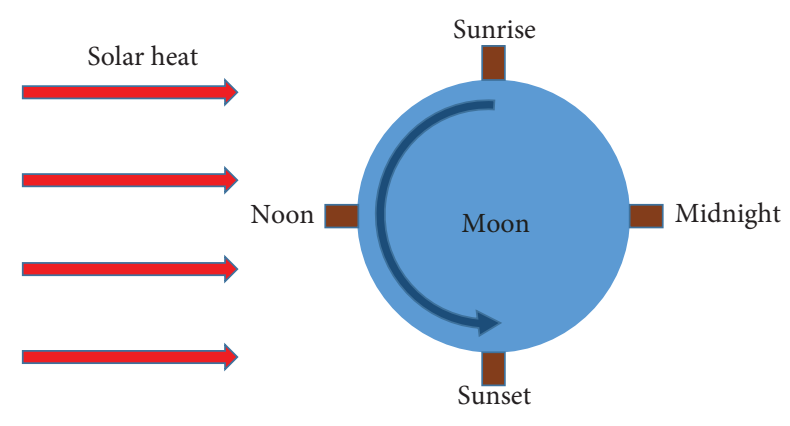

Figure 6: Location of lunar lander during 1 lunar day.

decrease in temperature due to the greater radiator area is negligible. This completes the design of the radiator area and the RHU. An excessive number of RHUs will increase the risk related to cost and mass while a too small number of RHUs will increase the risk related to thermal control requirements. Therefore, it is crucial to calculate the appropriate number of RHUs.

In this study, the lunar rover does not equip RHUs. The lunar rover moves into a shelter surrounded by an MLI curtain between the lunar lander and the lunar surface during lunar nights. The radiator of the lunar rover should be designed to ensure that the maximum temperature of the rover does not exceed the maximum acceptance temperature during lunar days.

The RHUs were evenly distributed inside the lunar lander, and heat load of the RHUs evenly distributed 20 watts on the 125 lander model nodes. Assuming that a single RHU weighs 40 grams and possesses a heat load of 0.9 watts [21], the lunar lander requires 22 RHUs with a total mass of $0.88 \mathrm{~kg}$ that can supply approximately $20 \mathrm{~W}$ of heat.

Table 5 shows the radiator area and the RHU capacity of the lunar lander and the rover derived from the aforementioned process.

\subsection{Temperature Analysis Results of the Lunar Lander and} the Rover. Figure 7 shows the calculated temperature contours of the lunar lander and the rover at the four locations defined in Figure 6. The temperature contours of the lunar lander and the rover shown in Figure 7 present the temperatures of the structures, excluding the MLI.

The solar panel was deployed after sunrise and stowed before sunset. The lunar rover moved outside the lunar lander after sunrise and returned inside the MLI curtain before sunset. In Figure 7, the temperature of lunar lander is the lowest at sunrise and the highest at noon. However, the lander reaches a maximum temperature between noon and sunset during a lunar day, as shown in Figure 8. The configuration of the deployed solar panel and the rover performing missions can be seen at the noon location in Figure 7. In addition, the temperature of the lunar regolith that was shadowed by the solar panel was observed to be lower than the temperature of the surrounding area due to the blocking of sunlight by the solar panel. At noon, the temperature of the regolith in the vicinity of the lunar lander was observed to be slightly higher than the temperature of the regolith further away from the lander. This is due to sunlight being reflected
TABLE 5: Thermal design result of the lunar lander and the rover.

\begin{tabular}{lc}
\hline Design category & Value \\
\hline Radiator area of the lander & $3600 \mathrm{~cm}^{2}$ \\
Radiator area of the rover & $640 \mathrm{~cm}^{2}$ \\
Heat capacity of the RHUs in the lander & $20 \mathrm{Watt}$ \\
\hline
\end{tabular}

from the MLI of the lunar lander, resulting in greater incident solar heat at the regolith near the lander compared to regolith further away from the lander. At sunset in Figure 7, the temperature at the shadow of the lander is lower than the temperature of the surrounding regolith, and the temperature of the regolith at the front end of the lander, which receives incident solar heat reflected by the lander, is higher compared to the surrounding regolith. The solar panel, which also acts as the lid of the lander radiator, receives little sunlight at sunset, which results in a substantially low temperature at sunset. When the solar panel (lid) covers the radiator of the lander, it begins to exchange heat with the lander. As a result, the temperature of the solar panel (lid) was confirmed to be higher at midnight compared to sunset. At midnight, the temperature of the regolith in the vicinity of the lander was observed to be slightly higher compared to the temperature of the surrounding regolith due to radiation heat exchange with the lander. The temperature of the regolith near the lander was observed to be lower at sunrise compared to midnight.

Figure 8 shows the temperature trend of the lunar lander during a lunar day. In Figure $8(\mathrm{a})$, the $x$-axis represents time, and the point at which the $x$ value is zero indicates sunrise. The point at which the $x$ value is 354.4 hours indicates sunset, at which the temperature abruptly drops due to the change in the heat dissipation of the lunar lander from 120 watts to 10 watts.

Figure 8(a) shows the temperature change at the center of the lander model, divided into five layers in the height direction, for a lunar day. The maximum temperature of the lander occurs at the bottom layer with a temperature of approximately $319 \mathrm{~K}$, which does not exceed the maximum temperature requirement of $50^{\circ} \mathrm{C}(323 \mathrm{~K})$ specified in Table 3. The minimum temperature of the lander occurs at the top layer and is approximately $257 \mathrm{~K}$. This also satisfies the minimum temperature requirement of $-20^{\circ} \mathrm{C}(253 \mathrm{~K})$ in Table 3. Therefore, results confirm that the temperature of the lander during a lunar day meets the acceptance temperature requirements in Table 3 .

When the lander enters lunar nighttime, the lid covers the radiator of the lander, and the heat loss is limited to a minimum. From this point, heat exchange takes place inside the lander for a certain period of time, and the temperature gradient decreases between the lander nodes. This period was termed the "convergence period," during which the temperatures of the lander nodes converge. The temperature trend during this period is shown in Figure 8(b). As shown in Figure 8(b), the temperature at the bottom of the lander, which possessed a relatively high temperature value, rapidly decreases due to the decrease in the heat dissipation value of the lander. However, the top of the lander, which possesses 


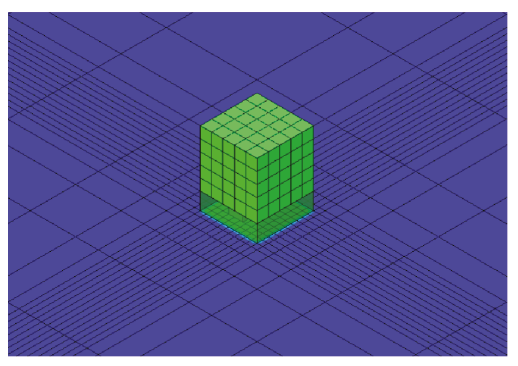

(a) Sunrise

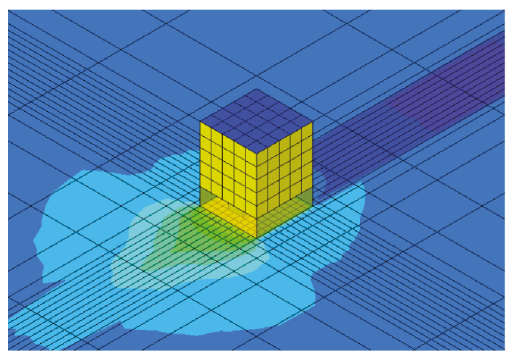

(c) Sunset

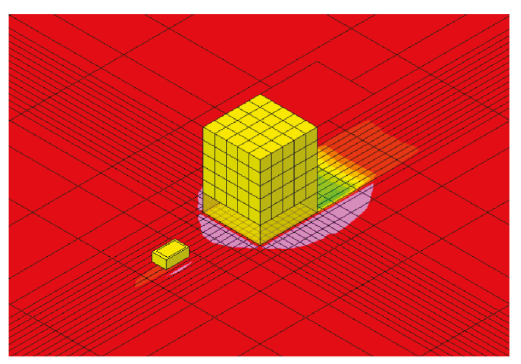

(b) Noon

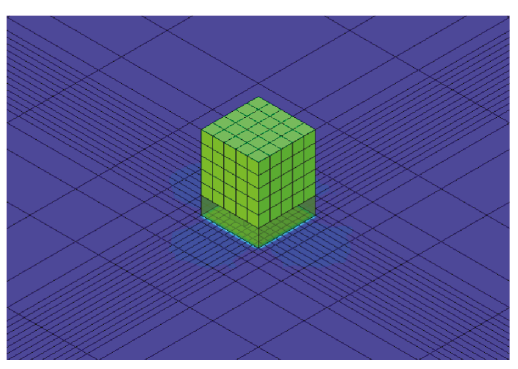

(d) Midnight

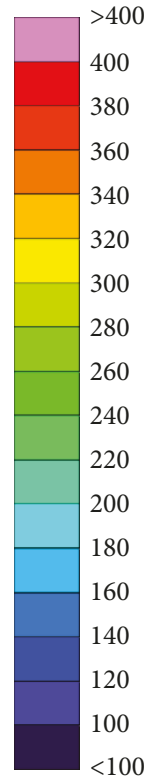

凷

Figure 7: Temperature contours of the lunar lander and the rover at each location.

a relatively low temperature, is temporarily warmed by the heat inflow from inside the lander. Once convergence is achieved over a period, the temperature of the entire lander decreases during the night.

The highest temperature of the lunar regolith occurs at noon, whereas the highest temperature of the lander occurs between noon and sunset. This is caused by the fact that, although the solar heat input is greatest at noon, the temperature of the lander is further increased past noon as the solar heat input is greater than heat rejection for a certain period. The time at which the temperature of the lander reaches a peak varies depending on the thermal capacity of the lander and the environmental heat exchange situation in the lander.

Figure 9 shows the average temperature trend of the lunar lander and the rover for a lunar day. In the case of actual rovers that perform lunar missions, a rover does not begin missions precisely at sunrise as it will need to receive solar power and produce electricity to perform its mission. However, in this study, a rover mission is assumed to take place outside the lander from sunrise to sunset to analyze the temperature behavior of the rover under the worst conditions.

Figure 9 shows that the temperature trend of the rover undergoes several inflections, unlike the temperature trend of the lander. This is due to the small thermal mass of the rover and the various temperature profiles influenced by environmental elements such as sunlight reflected from the lander. Similar to the lander, the rover reaches its highest temperature between noon and sunset due to the continuous input of solar heat even past noon until the rover reaches thermal equilibrium. Due to the relatively small thermal mass of the rover and the fact that the rover is affected by the temperature of the lander (which has a relatively large thermal mass), the temperature of the rover retains a flat shape for a certain period after reaching a peak temperature. This is in contrast to the case of the lander, where the temperature decreases immediately after the highest temperature. The temperature trend of the rover is affected by the relative position between the rover and the lander, and the shape of the graph in Figure 9 changes according to the changes in the relative position between the rover and the lander.

In Figure 9, as the rover moves through the MLI curtain into the shelter for lunar nights, the temperature of the rover rises for a certain period through heat exchange with the bottom of the lander, followed by a steady decrease in temperature as the lander temperature decreases. The temperature of the rover is maintained at a level slightly higher than the lander temperature during lunar nights due to heat from $0.5 \mathrm{~W}$ of standby power without heat exchange with the environment outside the lander. As a result, the maximum temperature of the rover is approximately $310 \mathrm{~K}$, which is below $45^{\circ} \mathrm{C}(318 \mathrm{~K})$, and the minimum temperature is approximately $263 \mathrm{~K}$, which is higher than $-15^{\circ} \mathrm{C}(258 \mathrm{~K})$. Therefore, the temperature of the rover during a lunar day meets the acceptance temperature requirement described in Table 3.

The temperature results of the lunar lander, shown in Figure 9, are similar to the results presented by Park et al. [6]. However, there are differences in the number of RHUs as well as the maximum and minimum temperatures of the lunar lander. This is due to the difference in the optical properties of OSR, but more so due to the fact that this study employed the CR-model [19] for MLI modeling, whereas Park et al. [6] used the $\varepsilon_{\text {eff }}$ model for MLI modeling.

In this study, the rover was able to satisfy the acceptance temperature requirement during lunar nights by simply moving into a sheltered location within the MLI curtain of the lander without mounting thermal control devices such as a lid or RHUs. The results confirm that, with assistance from an MLI curtain in the lunar lander, a small rover is able 


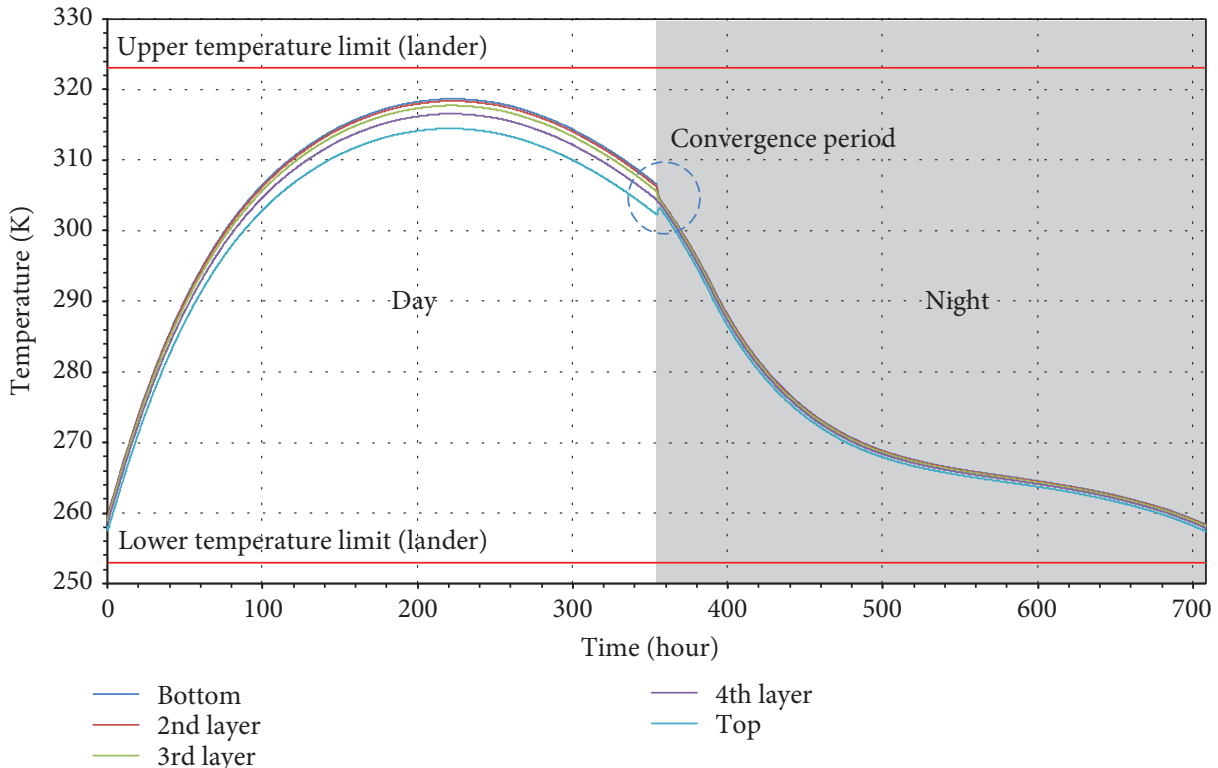

(a)

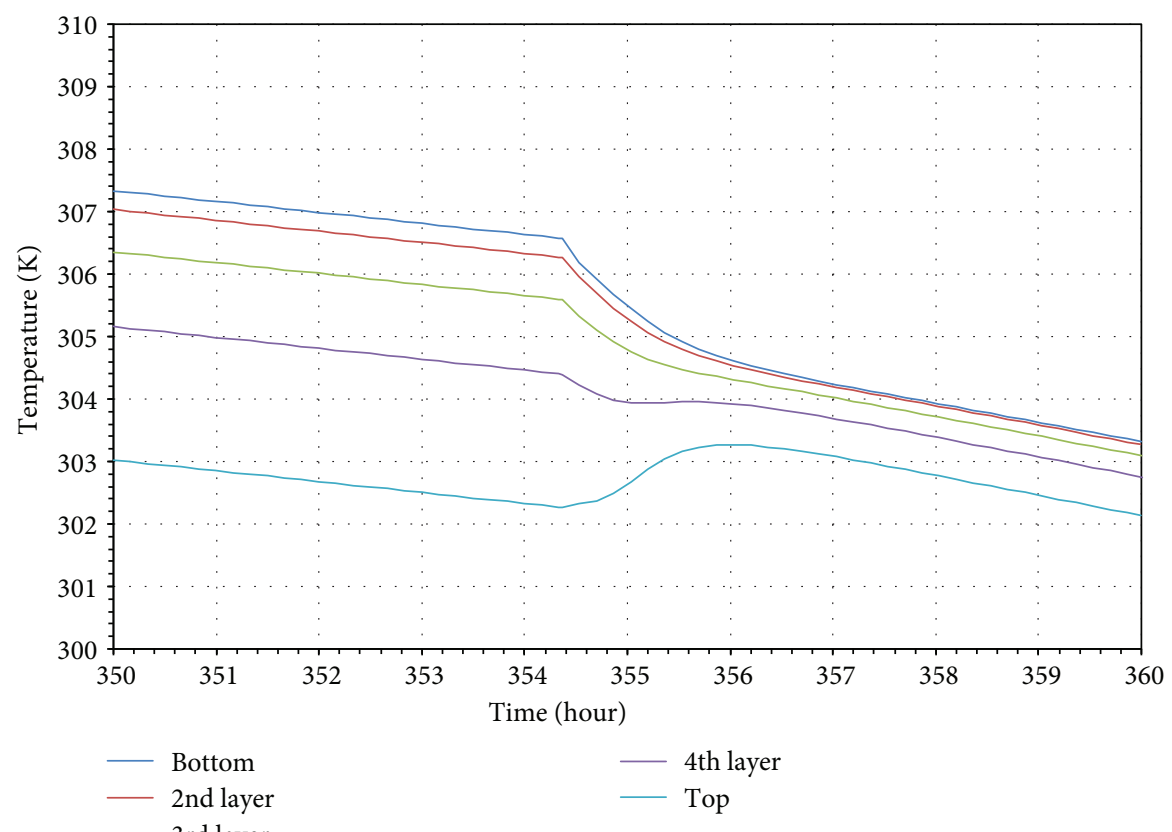

(b)

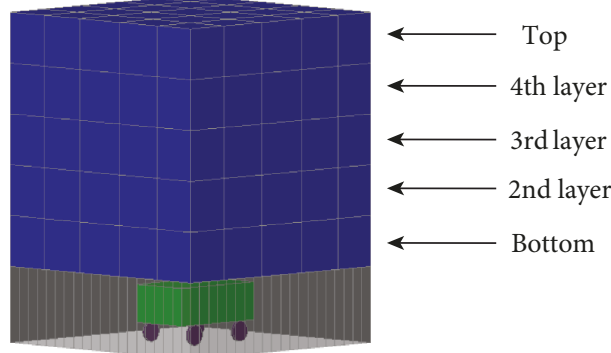

(c)

Figure 8: (a) Temperature trend of the lunar lander during a lunar day. (b) Temperature trend of the lunar lander during the convergence period. (c) Diagram of the lunar lander thermal model. 


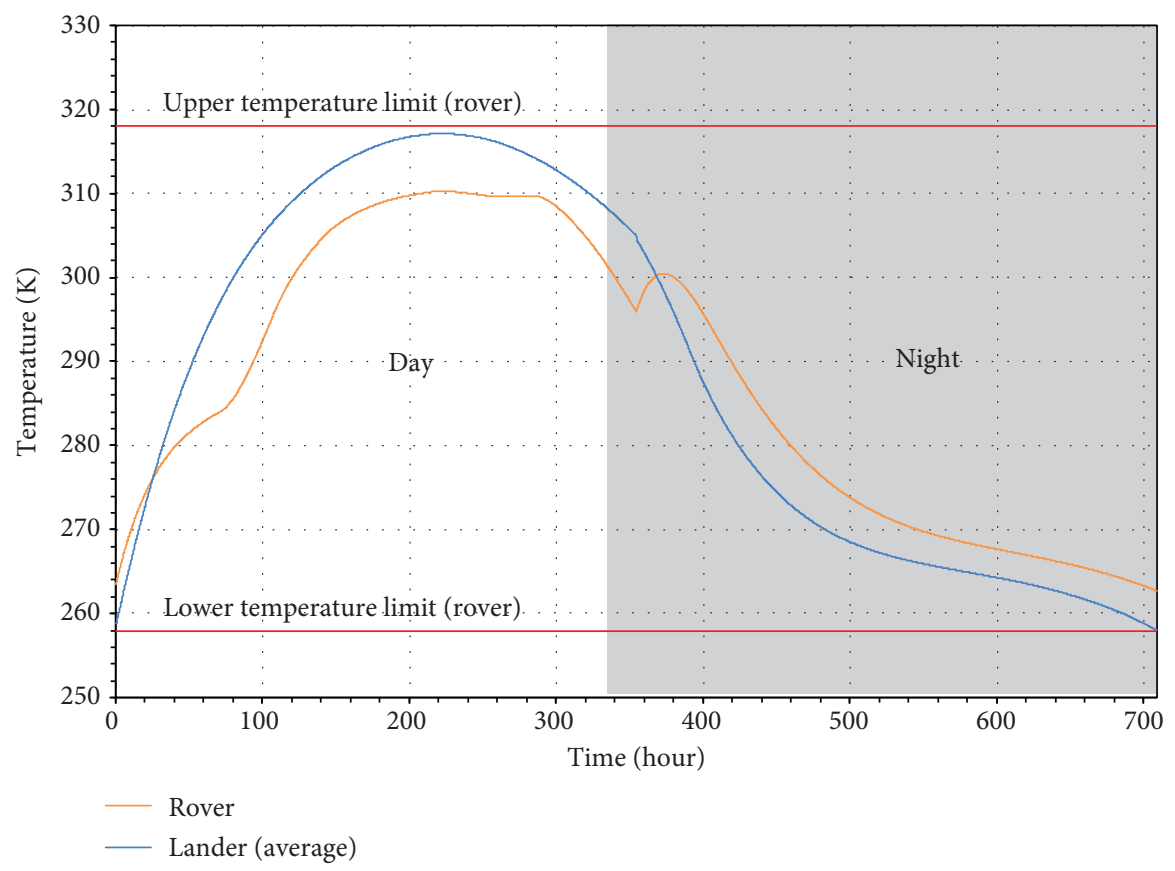

FIgURE 9: Average temperature trends of the lunar lander and the rover during 1 lunar day.

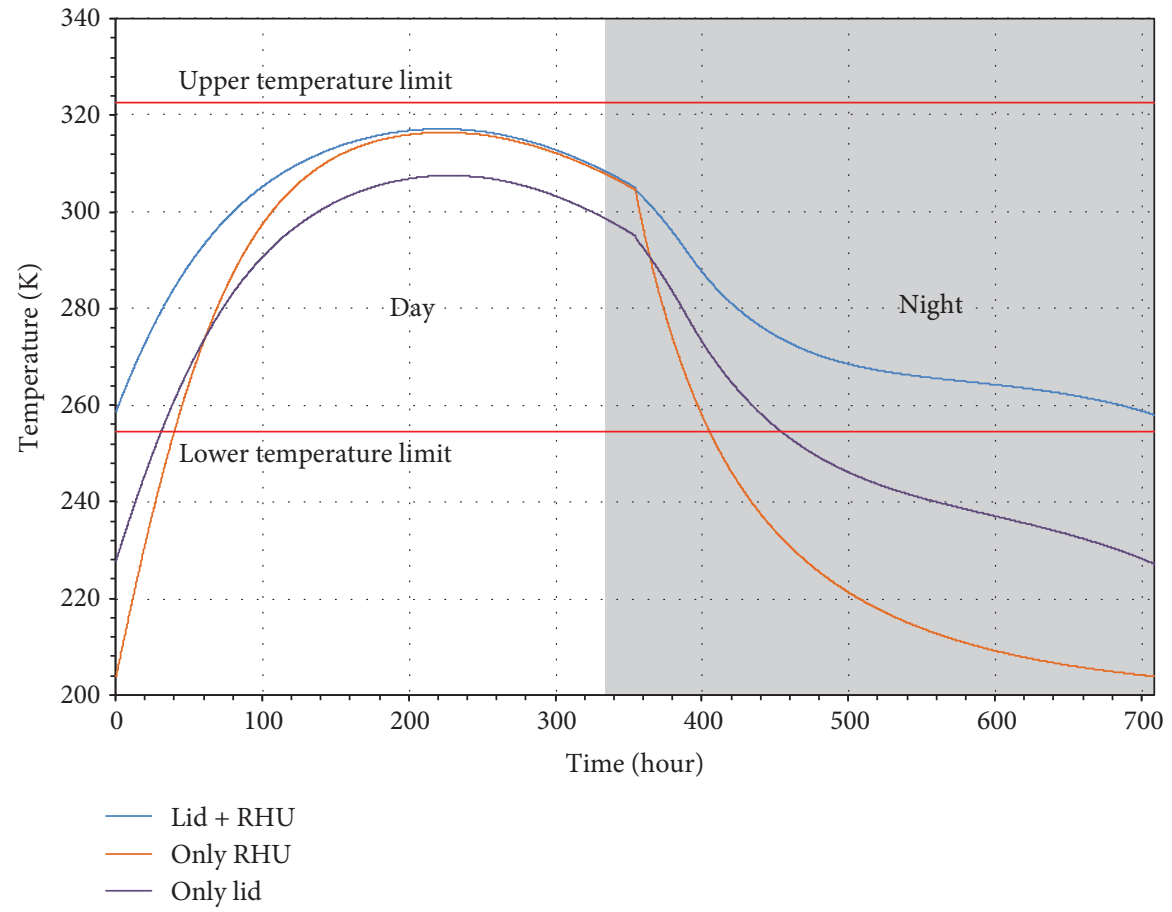

Figure 10: Case study on the efficiency of the lid and RHUs.

to carry out long-term missions for periods longer than a month, similar to large-sized rovers.

4.4. Analysis of the Influence of the RHUs and Lid. In order to examine the efficiency of the lid and RHUs of the lander, the temperature results of the lander without the lid and RHUs were compared with the thermal analysis results of the lander described in the previous section, as shown in Figure 10.
The important focal point of Figure 10 is the difference between the maximum and minimum temperatures during a lunar day. If the difference between the maximum and minimum temperatures is small, it is possible to satisfy acceptance temperature requirements even if the temperature results exceed the maximum or minimum acceptance temperatures by shifting the analysis temperature by adjusting the radiator area. In Figure 10, the difference between the 
maximum and minimum temperatures of the lander is approximately $59 \mathrm{~K}$, according to the analysis of the design results in Table 5 . The difference is approximately $80 \mathrm{~K}$ without the RHUs and approximately $113 \mathrm{~K}$ without the lid. Considering that the temperature difference of the acceptance temperature requirements is $70 \mathrm{~K}\left(-20^{\circ} \mathrm{C} \sim 50^{\circ} \mathrm{C}\right)$, it is difficult to achieve the acceptance temperature requirement without the RHUs and the lid even with adjustments in radiator area.

In the absence of a lid, the temperature drops rapidly after entering the lunar night, resulting in a larger difference between the maximum and minimum temperatures. Based on this result, an active device such as a lid is necessary for small lunar landers to survive lunar nights. Park et al. [6] also showed that lids are significantly more efficient compared to louvers or thermal switches. In Figure 10, the temperature decreases more rapidly in the absence of a lid compared to the absence of RHUs. Therefore, it can be concluded that, for a lunar lander, the effectiveness of a lid is greater than the effectiveness of RHUs for night survival.

For cases with a lid but no RHUs, the difference between the maximum and the minimum temperatures is $80 \mathrm{~K}$, which is greater than the requirement of $70 \mathrm{~K}$ of this study. However, the temperature requirement of the lunar lander may potentially be satisfied by efficiently arranging the internal components of an actual lunar lander or by optimizing the radiator area. However, this study could not make conclusions regarding this possibility as the lunar lander was modeled as a simple cube. Instead, a detailed study on this topic is suggested as a future research subject.

4.5. Regolith Temperature Analysis according to the Effect of the MLI Curtain. The temperature of the regolith inside the MLI curtain was compared to the temperature of the external regolith to investigate the heat protection effect of the MLI curtain of the lander on the lunar regolith. The results are presented in Figure 11.

In Figure 11, the temperature results of the bottom of the lander and the inner surface regolith were difficult to distinguish, as the temperature results were superimposed. Figure 11 shows that the temperature of the outer surface regolith is the same as the regolith temperature result of latitude $0^{\circ}$ introduced in Figure 3(a). The temperature of the inner surface regolith follows the temperature of the bottom of the lander. As the fluff layer of the lunar regolith possesses significantly low thermal conductivity and significantly small heat capacity, the temperature of the fluff layer inside the MLI curtain can be estimated to be similar to the temperature of the lander bottom which has a relatively large heat capacity.

Figure 11 shows that the surface temperature of the Moon outside the MLI curtain compared to the temperature within the MLI curtain is $71 \mathrm{~K}$ higher during the lunar day and $183 \mathrm{~K}$ lower during the lunar night. If a rover is forced to survive a lunar night outside the MLI curtain without survival equipment such as RHUs, the temperature of the rover is likely to exceed acceptance temperature requirements due to the harsh temperature conditions of the Moon. The MLI curtain idea is not feasible on planets like Earth, where there is an atmosphere and a surface with varying thermal conductivity and heat capacity depending on the location, and is only feasible in a heat environment like the Moon. As shown in Figure 11, the effectiveness of the MLI curtain was demonstrated by the substantial temperature difference between the space outside and inside the MLI curtain.

\section{Additional Suggestions}

5.1. Double Layer MLI Curtain. In this study, an MLI curtain was used to achieve lunar night survival for a rover. The MLI curtain provides shelter to the rover to prevent radiative heat exchange with the outside environment and to perform heat exchange with the lander. MLI curtains should also allow the rover to pass freely and possess the ability to completely block external views. In Section 2, this study recommended that the MLI curtain should be designed in the shape of blinds of several pieces. In order to prevent heat loss from gaps between the pieces of the blind shape, the MLI curtain could be designed with two layers and installed in a crosswise manner to enhance feasibility. Figure 12 shows the configuration of a double layer MLI curtain.

5.2. Rovers without Solar Cells. In this study, it was assumed that the rover performs missions by charging power through solar panels, as in the case of rovers that were launched in the past. Based on the concept of sheltering the rover within the MLI curtain during lunar nights, removing of the solar panels and SAR (Solar Array Regulator) devices from the rover and, instead, charging energy from the lander may be considered.

Rovers that convert solar energy into electrical energy are unable to perform missions during lunar nights. Conversely, rovers that perform missions by charging energy from a lander are able to perform lunar night missions, depending on the available capacity of the lander battery. In addition, rovers that are not equipped with solar panels and SAR devices weigh less, making it easier to meet mass requirements. Therefore, by increasing the capacity of the battery by a certain mass, a rover can successfully perform lunar night missions. However, without solar panels on the rover, there exists the restriction of having to return to the lander to charge the rover battery if the remaining energy of the rover battery is insufficient, even during lunar days.

A rover that employs a battery-charging method and performs missions during lunar nights have significant advantages, as it is possible to assign larger mission goals in terms of scientific inquiry. The technology that enables a small rover to recognize the position of the charging device that is equipped in the lander reflects the automatic charging technology of commercialized robot cleaners for home use, which is a technology that can be easily implemented.

\section{Conclusion}

This paper proposed a lunar night survival method of small rovers for long-term missions and verified the proposed idea through thermal analyses. Various types of landers and rovers that have landed on the Moon have been investigated, and long-term missions were identified as difficult tasks for 


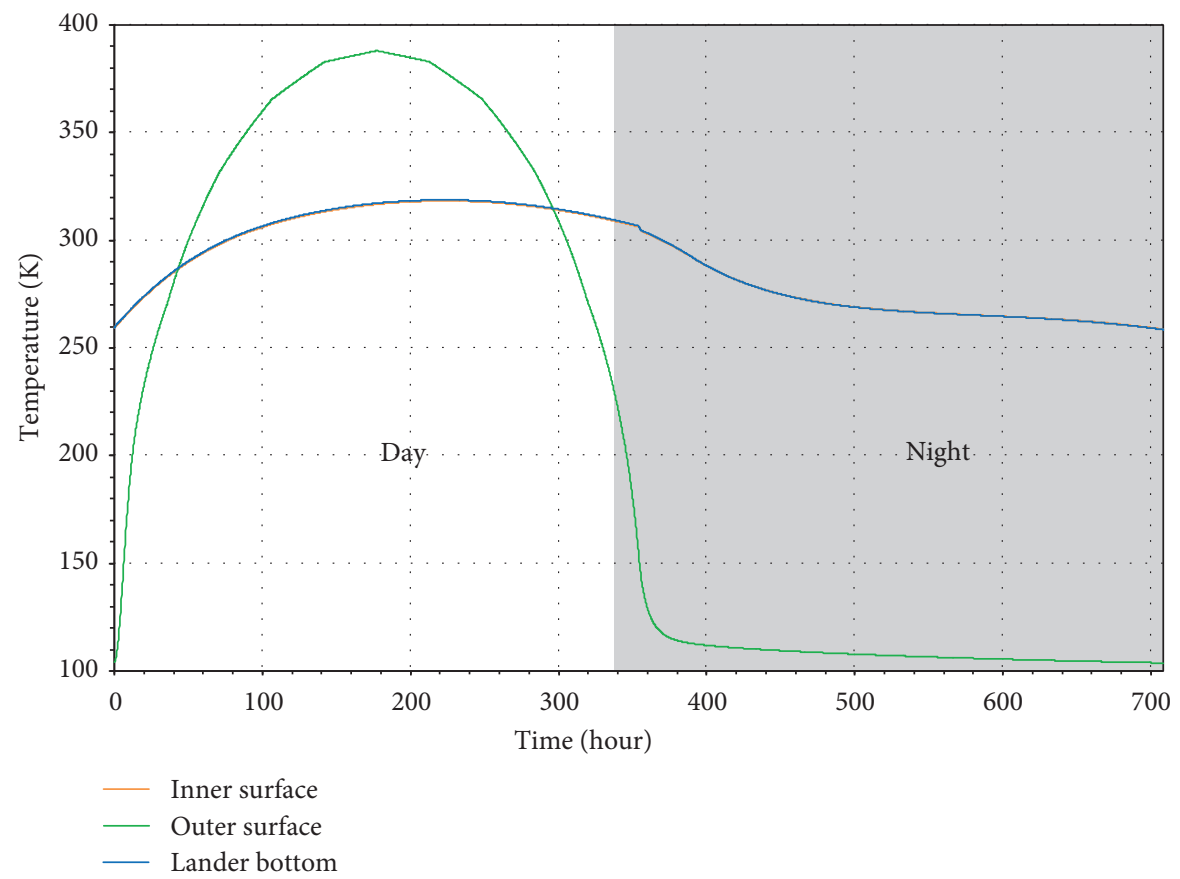

Figure 11: Comparison of the temperatures of the inner regolith and the outer regolith.

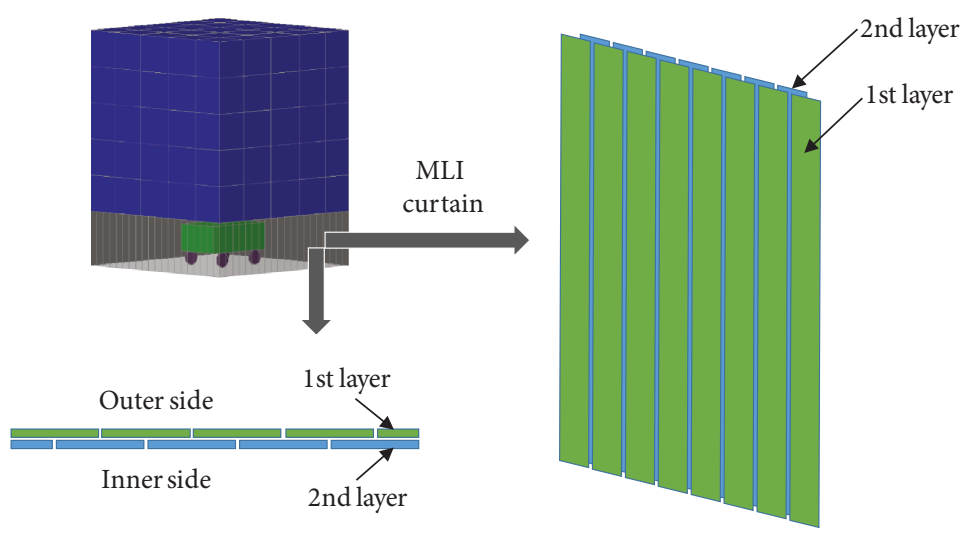

Figure 12: Concept configuration of a double layer MLI curtain.

small rovers. This study proposed a MLI curtain system for the night survival of small rovers. A lunar regolith thermal model was established and compared with LRO measurement results for verification. The lunar lander thermal model was modeled with a simple cubic shape, and RHUs and a lid were combined as thermal designs to ensure that the lander could survive the lunar thermal environment and meet the acceptance temperature requirements. As a result, this study derived the optimized thermal design for a lander to survive lunar days and nights. A thermal model of the MLI curtain and the rover was developed, which is a key proposal of this study. Thermal analysis showed that the proposed night survival system involving an MLI curtain enables a small lunar rover to survive lunar nights without RHUs or other temperature maintenance devices.

The lunar lander and the rover were modeled as simple cubes in this study. Therefore, it is suggested for future stud- ies to verify the details presented in this study using detailed thermal models of lunar landers and rovers that simulate electrical boxes and internal radiation heat exchanges. With further research on rovers that are not equipped with solar panels and instead supplied with power solely by a lander battery, lunar exploration missions can be advanced further to enable wider research areas such as lunar night periods.

\section{Data Availability}

The data used to support the findings of this study are available from the corresponding author upon request.

\section{Conflicts of Interest}

The authors declare that they have no competing interests. 


\section{Acknowledgments}

This research was financially supported by the Korean Lunar Exploration Program of the National Research Foundation of Korea (NRF) funded by the Ministry of Science and ICT (MSIT) (NRF-2016M1A3A9005561).

\section{Supplementary Materials}

This file includes the raw data of the charts presented in the manuscript. (Supplementary Materials)

\section{References}

[1] L. Summerer, Technical Aspects of Space Nuclear Power Sources VII. Radioisotope Heater Units, European Space Agency, ACT-RPT-2327-RHU, 2006.

[2] R. Balasubramaniam, R. S. Wegeng, S. A. Gokoglu, N. H. Suzuki, and K. R. Sacksteder, Analysis of solar-heated thermal wadis to support extended-duration lunar exploration, NASA/TM-2010-216254, 2010.

[3] R. Notsu, H. Nagano, and H. Ogawa, "Conceptual verification of lunar long-duration method by using high-heat-storagecapability of regolith," Journal of Thermophysics and Heat Transfer, vol. 29, no. 1, pp. 65-73, 2015.

[4] http://pib.nic.in/newsite/PrintRelease.aspx?relid=183103.

[5] S. Okishio, H. Nagano, and H. Ogawa, "A proposal and verification of the lunar overnight method by promoting the heat exchange with regolith," Applied Thermal Engineering, vol. 91, pp. 1176-1186, 2015.

[6] T.-Y. Park, J.-J. Lee, J.-H. Kim, and H.-U. Oh, "Preliminary thermal design and analysis of lunar lander for night survival," International Journal of Aerospace Engineering, vol. 2018, Article ID 4236396, 13 pages, 2018.

[7] S. Kassel, Lunokhod-1 Soviet Lunar Surface Vehicle, Advanced Research Projects Agency, 1971.

[8] L. B. Gary, J. L. James, J. H. Richard et al., "Mission of daring: the general-purpose heat source radioisotope thermoelectric generator," in 4th International Energy Conversion Engineering Conference and Exhibit (IECEC), San Diego, CA, USA, June 2006.

[9] A. R. Vasavada, D. A. Paige, and S. E. Wood, "Near-surface temperatures on Mercury and the Moon and the stability of polar ice deposits," Icarus, vol. 141, no. 2, pp. 179-193, 1999.

[10] P. B. Hager, Dynamic thermal modeling for moving objects on the Moon, Technische Universität München, München, 2013.

[11] R. J. Christie, D. W. Plachta, and M. M. Hasan, Transient Thermal Model and Analysis of the Lunar Surface and Regolith for Cryogenic Fluid Storage, NASA/TM-2008-215300, Glenn Research Center, Cleveland, OH, USA, 2008.

[12] A. R. Vasavada, J. L. Bandfield, B. T. Greenhagen et al., "Lunar equatorial surface temperatures and regolith properties from the Diviner Lunar Radiometer Experiment," Journal of Geophysical Research, vol. 117, article E00H18, pp. 1-12, 2012.

[13] C \& R Technologies, Thermal Desktop User's Manual, Ver 5.8, C \& R Technologies, Inc., Boulder, CO, USA, 2017.

[14] J.-P. Williams, D. A. Paige, B. T. Greenhagen, and E. SeftonNash, "The global surface temperatures of the moon as measured by the Diviner Lunar Radiometer Experiment," Icarus, vol. 283, pp. 300-325, 2017.

[15] https://www.bobthealien.co.uk/moon/landingsites.htm.
[16] D. G. Gilmore, Spacecraft Thermal Control Handbook, Volume1: Fundamental Technologies, The Aerospace Corporation, El Segundo, CA, USA, 2nd edition, 2002.

[17] B. McKissock, P. Loyselle, and E. Vogel, Guidelines on Lithium-ion Battery Use in Space Applications, NASA/TM2009-215751, Glenn Research Center, Cleveland, OH, USA, 2009.

[18] https://www.hq.nasa.gov/alsj/LM_Lunar_Module_ppLV117.pdf.

[19] C. K. Krishnaprakas, K. Badari Narayana, and P. Dutta, "Heat transfer correlations for multilayer insulation systems," Cryogenics, vol. 40, no. 7, pp. 431-435, 2000.

[20] https://www.qioptiq.com.

[21] https://rps.nasa.gov/system/downloadable_items/31_Final_ RHU_Fact_Sheet_2016_5-26-16.pdf.

[22] https://nssdc.gsfc.nasa.gov/nmc/SpacecraftQuery.jsp.

[23] https://www.nasaspaceflight.com/2019/01/china-returningmoon-change-4-mission/.

[24] https://www.airspacemag.com/daily-planet/chinas-journeylunar-far-side-missed-opportunity-180963703/.

[25] S. Amitabh, T. P. Suresh, and K. Srinivasan, "Potential landing sites for Chandrayaan-2 lander in southern hemisphere of Moon," in 49th Lunar and Planetary Science Conference, The Woodlands, TX, USA, March 2018.

[26] https://www.lpi.usra.edu/sbag/meetings/jan2014/presentations/ 08_1545_McNutt_Pu238_SBAG.pdf. 


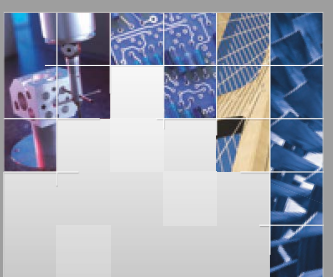

\section{Enfincering}
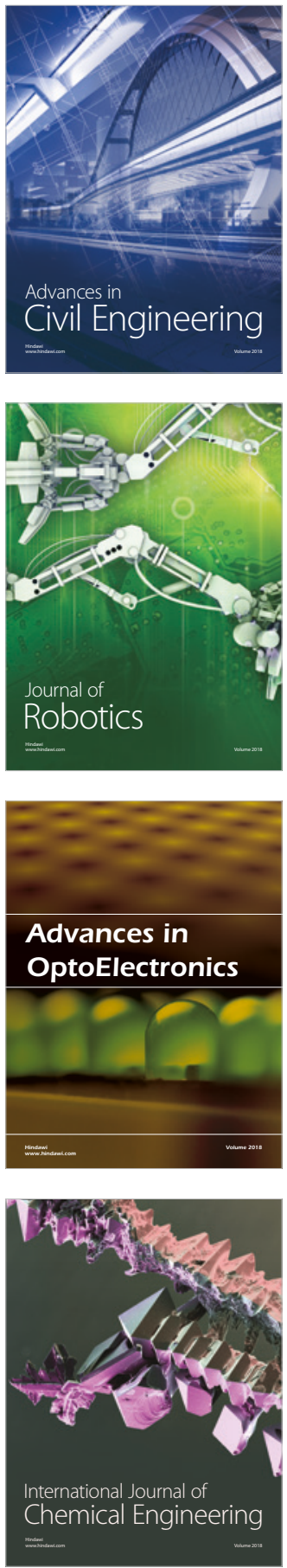

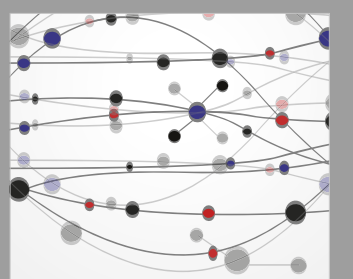

\section{Rotating \\ Machinery}

The Scientific World Journal

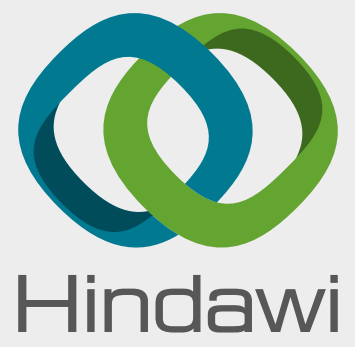

Submit your manuscripts at

www.hindawi.com
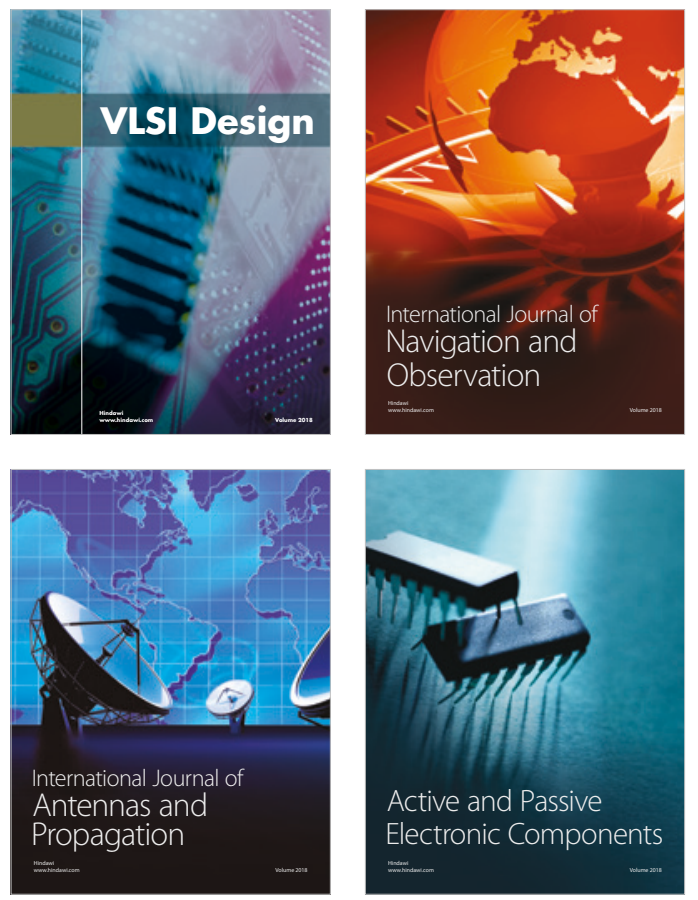
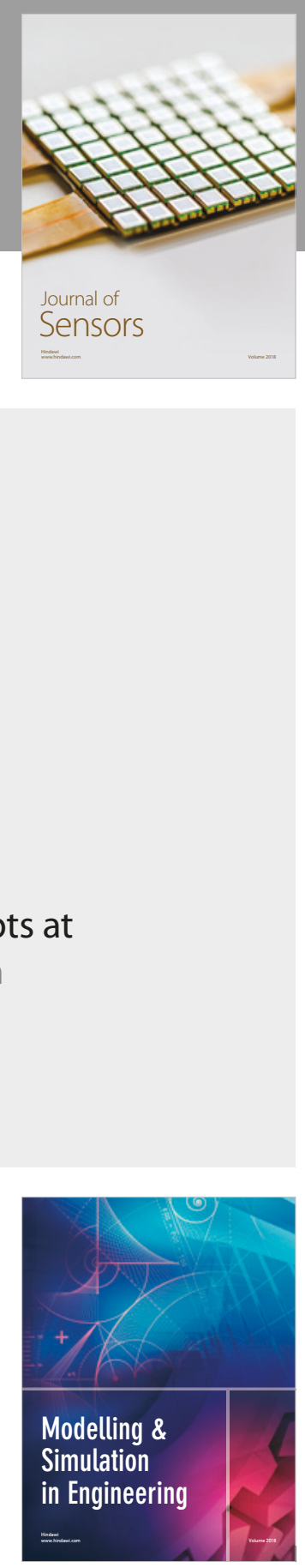

\section{Advances \\ Multimedia}
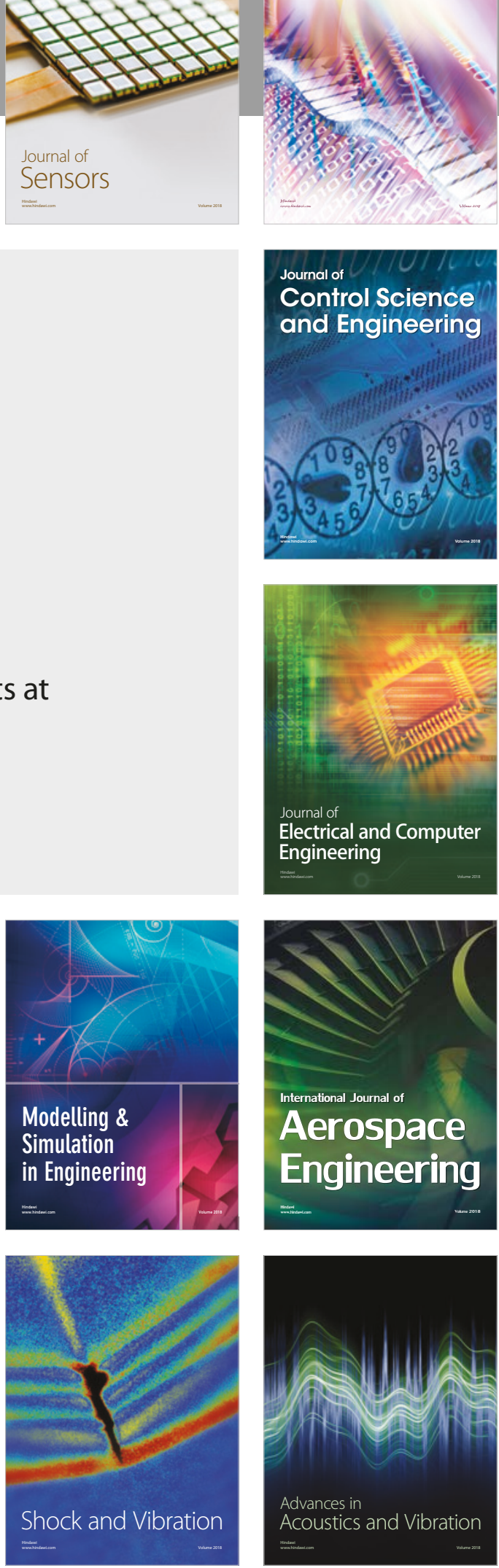